\title{
A Spectral Approach for the Efficient Identification of Power Transmission Network Uncontrolled Separation
}

\author{
Youwei Jia, ${ }^{1,3}$ Chun Sing Lai $\odot,{ }^{2}$ and Zhao Xu $\odot^{1,3}$ \\ ${ }^{1}$ Department of Electrical Engineering, The Hong Kong Polytechnic University, Hong Kong, P. R. China \\ ${ }^{2}$ Energy and Power Group, Department of Engineering Science, University of Oxford, Oxford, UK \\ ${ }^{3}$ School of Electrical and Information Engineering, Changsha University of Science and Technology
}

\section{CONTENTS}

1. Introduction

2. Related Study

3. The Proposed Methodology

4. Case Studies

5. Conclusions

Funding

References
Keywords: uncontrolled separation, operation security, cascading failure, spectral clustering, transmission network

Address correspondence Zhao Xu, Department of Electrical Engineering, The Hong Kong Polytechnic University, Hung Hom, Hong Kong, P. R. China.

E-mail: eezhaoxu@polyu.edu.hk

\begin{abstract}
Uncontrolled network separation (system island formation) is one of the most critical contingencies in power systems. The integrity of the whole power transmission network is a prerequisite for reliable system operation. Therefore, in order to safeguard the system operation and control, it is imperative to quickly identify the topological changes, especially the formation of system islands. In this paper, we developed a spectral clustering-based approach to efficiently detect the existing or the potential network islands. The core of this approach is a graph-algebraic model, which combines the real-power deliverability and topological information of a power transmission network. Based on an improved spectral clustering algorithm, the proposed approach can efficiently identify the critical situations of the network splitting under multiple line outages. This approach has been successfully tested by using the New England 39-bus and 118-bus systems under different scenarios.
\end{abstract}

\section{INTRODUCTION}

Being a critical infrastructure in the modern society, the power system is considered as a "lifeline system" in the present day. Its operation security and reliability have significant influences on all aspects of human life. In many countries, it has been noted that the power grids are driven to stressful operating points, which are near to their intrinsic limits. This can be due to the fact that the existing power transmission systems fail to fully accommodate the increasing load demands. Moreover, the power system operation is continuously subject to various internal or external disturbances, such as malfunctions of protection devices (e.g., hidden failures of relays), faulty manipulations of operators, incidents caused by human or animals, and environmental disasters. Such disturbances can cause transmission line outages that would deteriorate the connectivity of the entire network. In the worst case, multiple outages can split the network into isolated components and the system ceases to operate as a whole. Generally, the loss of system integrity would result in serious active and reactive 
issues, such as voltage collapse, frequency instability [1], and so forth.

The benefits of timely identifying the formation of network separations are twofold for the power system operation. First, it provides indispensable information for off-line simulations. If a network splits, all the steady-state analysis programs (e.g., state estimation) that use Newton power flow methods would suffer from the singularity of Jacobian matrix [2]. The inefficient modification of Jacobian matrix would lead to a great discrepancy between the actual power flows and the off-line results. Second, the effective islanding detection allows suitable control strategies to be employed to counteract the extreme events. For example, with a prior knowledge of network separations, an effective dispatching scheme can be deployed in a timely manner to manage the sources in different areas, so that the generation-load balance can be maintained to a great extent.

In a large interconnected power transmission network, frequency stability would be of concern following a severe system disturbance, resulting in unexpected cascading outages leading to splitting of the network into two or more islands (i.e., sub-networks). Frequently, such unexpected splitting would lead the system to an escalation phase of cascading failures followed by widespread blackouts. In this regard, North American reliability standards now require that "Each transmission operator shall operate to protect against instability, uncontrolled separation, or cascading outages resulting from multiple outages." The motivation in the present work is to timely identify the existing and potential uncontrolled network separations. In general, such an identification is not visually straightforward for system operators due to the high complexity of the network structure. Network-splitting patterns are usually "hidden" in the sense that they do not manifest at the early stage until some severe contingencies expose their existence.

Based on the steady-state analysis, the network separation problems are generally resolved by using a numerical analysis or graph-theoretic methods. Refs. [3, 4] present the pioneering works on islanding detections in power systems based on the monitoring data and linked list tables. In Refs. $[1,2,5]$, some numerical approaches are reported based on network nodal connectivity matrix, LU decomposition, and eigenevaluation of susceptance matrix, respectively. Graph-theoretic methods are stemmed from graph theory and focus on the relations of nodes and edges. In Ref. [6], node fusion is applied to an outage analysis in the infrastructure network. A breadth-first search algorithm utilized in the electric networks is developed in Ref. [7]. In Ref. [8], an effective approach based on the path search is proposed. In general, numerical approaches are computationally efficient but fail to address the topological properties of the practical networks. In contrast, existing graph-theoretic methods based on a global search take into account the topological properties while entailing high computation costs.

In this paper, the network separation patterns are revealed and analyzed in a spectral domain through the proposed approach, which turns out to be effective and efficient in medium-scale transmission networks. It should be noted that the real-power imbalance-induced cascading outages and the topological changes of power transmission network are the main concerns in the present work. Although the system dynamics, voltage stability, and other causal factors of the network separation (e.g., environmental disasters) are of interest in their own right, they require much more comprehensive modeling and some of them still remain qualitative or conceptual at this stage. Hence, they will be considered in our future work and not discussed further in this paper.

The remainder of the paper is organized as follows. Section 2 briefly reviews the existing research on complex network modeling employed in the power system analysis and graph-partitioning algorithms. Section 3 introduces the proposed approach for the identification of network separations in a static sense. Case studies are carried out and simulation results shown in Section 4 demonstrate the effectiveness of the proposed approach. Section 5 concludes this paper.

\section{RELATED STUDY}

Incorporating complex network theories into the power system analysis has provided novel and useful solutions to many practical issues [9]. Initially, physicists developed complex network theories such as Erdos-Renyi random networks to mathematically analyze the structure and linkage of networks for their research on abstract networks [10-12]. Due to the abstract nature, the classical complex network is unable to fully address the physical properties of many practical networks. Motter et al. studied the Internet and power system networks and discovered the robust-but-fragile feature under cascade-based attacks $[13,14]$. Simultaneously, some complex network models were reported to investigate the structural vulnerability of the power grids [9, 15]. These works mainly focus on the physical topologies and structural connection of the networks, while mostly neglect the specific structural properties and interactions between the network components governed by engineering principles.

The power transmission network design aims at transferring the electrical power from the generation to the demand side. The functionalities of power transmission network are significantly dependent on the topological structure, which is greatly affected by their organizational complexities and 
interplays among all electrical components [16]. In Ref. [17], Barabasi-Albert discovered that the scale-free network, which is characterized by heterogeneous structures and non-uniform degree distributions, possesses some important features such as the robustness against random failures as well as vulnerability to the attacks on their hubs, i.e., nodes with high degree and high connectivity. Once the network is changed by, for example, component outages, the network functionality may be significantly weakened. Recently, specific features and interplays of electric components in the power transmission networks have been studied in Refs. [18-20] from a complex network perspective, which deserves further research efforts.

To the authors' best knowledge, there have been few applications of complex networks to tackle the challenging issues of uncontrolled network separation in power systems. Previously, some research works revealed that the large and highly interconnected power systems contain small-world features, where different parts are highly interdependent on each other and interact directly or indirectly. One particular property of a small-world network is that the network distance or connectivity can be greatly affected by the topological changes [21]. One failure in a part may notably propagate to other parts, followed by splitting or even causing the whole network to collapse. Preceding research works are fairly valuable for the system islanding detection, while those works are principally based on the qualitative analysis.

Naturally, detecting system separation in the power transmission networks is closely related to the graph-partitioning research, where many relevant algorithms and approaches have been developed thus far. As a pioneering work, Girvan and Newman proposed an iterative method based on the concept of betweenness [22-24], which can well recognize the network-splitting patterns by recursively removing edges with the largest betweenness. This approach yields very good partitioning results but is computationally heavy and time-consuming. In Ref. [25], a divisive algorithm is reported that can well handle triangular or higher order arcs, i.e., loops exposed in networks. As proposed in Ref. [26], the Wu-Huberman algorithm is based on the idea of voltage drop, which has a short computational time but still involves iterative computing. Based on the q-state Potts Hamiltonian, the Reichard-Bornholdt method is reported in Ref. [27], which is a pioneering approach for identifying fuzzy communities. In Ref. [28], the Capocci-ServedioColaiori-Caldarelli method combines spectral properties of networks with correlation measurements to detect the closeness of communities. For further reading of aforementioned methods, a comprehensive review is presented in Ref. [24]
By applying the graph-partitioning algorithm to islanding detection in power transmission networks, we accentuate the need of computing efficiency and accuracy. For this reason, a tailor-made improved spectral clustering algorithm is proposed in this paper to capture the insight into emerging topological separation.

\section{THE PROPOSED METHODOLOGY}

\subsection{Complex Network Modeling of Power Transmission Networks}

A power transmission network consists of generation buses, load buses (i.e., demand sides), electrical facilities (e.g., transformers, relays, breakers, etc.), and physical links (i.e., transmission lines). A secure and reliable operation of power transmission networks aims to transfer electric power from generators to different loads. Based on the concept of complex network, the power transmission networks can be abstracted into directed or non-directed graphs with $n$ vertices and $m$ edges in different research cases. In order to obtain a general solution of islanding detection, a power transmission network is regarded as a non-directed graph in this paper, where generators are considered as source vertices, loads are considered as sink vertices, and transmission lines and transformers are considered as weighted edges. This is reasonable as bi-directional power flows exist in most equipment of power transmission networks such as transmission lines. Mathematically, a power transmission network can be denoted by $G(N, E)$, where $N=\left\{n_{1}, n_{2}, \ldots, n_{|N|}\right\}$ is the set of all vertices and $E=\left\{e_{1}, e_{2}, \ldots, e_{|E|}\right\}$ is the set of all edges. Each edge corresponds to a vertex pair $\left(n_{i}, n_{j}\right)$.

In power system, the nodal injection power is calculated as follows:

$$
\left.\begin{array}{rl}
P_{i} & =V_{i} \sum_{j \in i} V_{j}\left(G_{i j} \cos \theta_{i j}+B_{i j} \sin \theta_{i j}\right) \\
Q_{i} & =V_{i} \sum_{j \in i} V_{j}\left(G_{i j} \sin \theta_{i j}-B_{i j} \cos \theta_{i j}\right)
\end{array}\right\}
$$

where $P_{i}$ and $Q_{i}$ represent the active and reactive injection powers of node $i$, respectively, and $V_{i}$ is the bus voltage of node $i$.

The incremental active and reactive powers can be formulated as follows [29]:

$$
\left[\begin{array}{c}
\Delta P \\
\Delta Q
\end{array}\right]=\left[\begin{array}{cc}
H & N \\
J & L
\end{array}\right]\left[\begin{array}{c}
\Delta \theta \\
\Delta V / V
\end{array}\right]
$$

In a high-voltage power system (i.e., transmission system), real-power flow is mainly subjected to the bus angle, while 
reactive power is mainly subjected to the bus voltage. Therefore, Eq. (2) can be simplified as follows:

$$
\left.\begin{array}{c}
\Delta P=H \cdot \Delta \theta \\
\Delta Q=L \cdot \Delta V / V
\end{array}\right\}
$$

$\boldsymbol{H}$ and $\boldsymbol{L}$ can be specified as follows:

$$
\begin{aligned}
H=L & =\left[\begin{array}{llll}
V_{1} & & & 0 \\
& V_{2} & & \\
& & \ddots & \\
& & & V_{n}
\end{array}\right]\left[\begin{array}{cccc}
B_{11} & B_{12} & \cdots & B_{1 n} \\
B_{21} & B_{22} & & B_{2 n} \\
\vdots & \vdots & \ddots & \vdots \\
B_{n 1} & B_{n 2} & \ldots & B_{n n}
\end{array}\right] \\
& \times\left[\begin{array}{llll}
V_{1} & & & 0 \\
& V_{2} & & \\
& & \ddots & \\
0 & & & V_{n}
\end{array}\right]
\end{aligned}
$$

where $B_{i j}$ is the entry of susceptance matrix of power system.

According to $\boldsymbol{H}$ matrix, $h_{i, j}(i \neq j)$ not only indicates the topological connection of power system, but also reflects the power transmission capability of each edge. Based on the classical concept of adjacency matrix, a new and symmetric matrix, termed as the power adjacency matrix (denoted as $A_{p}$ ), is proposed to generalize the topological and electrical features of power transmission networks.

Definition 1: The set of real numbers is denoted by $R . A_{p} \in$ $|\mathrm{N}| \times|\mathrm{N}|$ consists of off-diagonal elements of $\boldsymbol{H}$ obtained by the Newton-Raphson power flow solution. Each entry of $A_{p}$ is expressed as follows:

$$
a_{i, j}= \begin{cases}h_{i, j} & i \neq j \\ 0 & i=j\end{cases}
$$

Based on Definition 1, the degree matrix $\boldsymbol{D}$ is redefined as follows:

$$
D(G)=\operatorname{diag}\left\{d_{1}, d_{2}, \ldots, d_{|\mathrm{N}|}\right\}, \quad \text { and } d_{i}=\sum_{j \in N} a_{i, j}, i \in N
$$

Based on the graph theory, the Laplacian matrix is viewed as a matrix representation of a graph. In the sense of classical definition of Laplacian matrix and special features of power transmission networks, the electric-Laplacian matrix (denoted as $L_{e}$ ) is proposed, which contains the topological and electrical information of power transmission networks.

Definition 2: Given a power transmission network, which consists of $n$ buses and $m$ transmission lines, $L_{e}$ is defined as follows:

$$
L_{e}=D-A_{p}
$$

where the diagonal element of $L_{e}$ is given by the degree of associated vertex in the power transmission network, and the off-diagonal element indicates the electrical attribute of each corresponding edge. According to the basic definition of Laplacian matrix [30], $L_{e}$ is symmetric, singular, and positive semidefinite.

\subsection{Spectral Properties of $\mathbf{L}_{\mathbf{e}}$}

Various disturbing events (e.g., multiple transmission line outages) would deteriorate the connectivity of a power transmission network and lead the power system to an escalation phase of cascading failures, which would ultimately cause widespread blackout. Based on some global search algorithms, identifying the network separation is an NP-hard problem. In addition, such algorithms are less computationally efficient and are difficult to identify the arisen islanding situations in terms of topological changes. In the graph theory, spectral partitioning algorithms provide an efficient and systematic solution to study the connection patterns of a graph. Some previous studies on spectral properties of a graph are reported in Refs. [28, 31]. Inspired by these works, we proposed a useful method based on normalized spectral clustering with a proper extension to fit with specific cases of power system uncontrolled separation. In this subsection, spectral properties of electrical Laplacian are investigated in detail. Some relevant theorems and proposition are introduced to address the relationship between the network topological changes and the Laplacian spectrum.

Notation: The first $k$ eigenvectors of $L_{e}$ are denoted by $v_{l}$, $v_{2}, \ldots, v_{k}$. The eigenvalues of $L_{e}$ are denoted by $\lambda_{i}, i=1,2$, $\ldots,|\mathrm{N}|$. We write $\lambda_{1} \leq \lambda_{2} \leq \lambda_{3} \ldots \leq \lambda_{|N|}$ to be the ascending order of eigenvalues of $L_{e} . S \in{ }^{|\mathrm{N}| \times k}$ contains the first $k$ eigenvectors as columns. $x_{i}$ is denoted as the $i$ th entry of a eigenvector $v_{i}$.

Theorem 1: [32-34]: If $G$ is a given weighted graph with all weights non-negative, the following statements are true:

(i) The Laplacian matrix of $G$ has only real eigenvalues.

(ii) The smallest eigenvalue $\lambda_{I}$ is equal to 0 and its corresponding eigenvector is constant, i.e., $(1,1, \ldots 1)^{\mathrm{T}}$. The multiplicity of zero eigenvalues is determined by the number of splitting sub-graphs of $G$.

(iii) $\lambda_{1}=0$ and $\lambda_{2}>0$ if $G$ is connected.

This theorem is derived from the Perron-Frobenius theorem, and the proof can be found in Refs. [33-35].

The second smallest Laplacian eigenvalue $\lambda_{2}$ is generally termed as algebraic connectivity of $G$, which reflects significant topological information of the network. Based on 
Inputs: $k$ is the number of clusters.

orig_c is the original set of centroids obtained by the existing control areas in a power transmission network. ang_dis1 is the set of angular distance of all points with reference vector $(1,0)$.

ang_dis2 is the set of angular distance of all points with reference vector $(-1$, $\sqrt{3})$.

ang_dis3 is the set of angular distance of all points with reference vector $(-1,-\sqrt{3})$.

Output: all clusters and corresponding centroids

Function: Clustering

Initialization by orig_c;

For each ang_dis,

Solve ("kmeans"is a MATLAB function):

$[i d x$, centroid,sumd $]=k m e a n s$ (ang_disn, $k$, 'emptyactio $n$ ', 'singleton', 'distance', 'sqEuclidean);

Repeat this process several times until stable solutions are reached;

End for

Select the solution obtained by ang_disn with minimum sumd;

FIGURE 1. Improved k-means algorithm.

Courant-Fischer's theorem [30], the second smallest eigenvalue (non-vanishing) can be formulated as follows:

$$
\lambda_{2}=\min v_{i}{ }^{T} \cdot L_{e} \cdot v_{i}, i=1,2, \ldots,|\mathrm{N}|
$$

To address how the Laplacian spectrum changes along with subjoining or subtracting an edge in the network, the CourantWeyl inequality [35] is introduced as follows:

Theorem 2: Let $\mathrm{G}^{*}=\mathrm{G}+$ ebe a graph reformed by inserting a new edge e into $G$. $\mathrm{G}^{*}$ and $\mathrm{G}$ have the same set of vertices. The following inequality is true:

$$
\begin{aligned}
0= & \lambda_{1}(G)=\lambda_{1}\left(G^{*}\right) \leq \lambda_{2}(G) \leq \lambda_{2}\left(G^{*}\right) \leq \lambda_{3}(G) \leq \cdots \\
& \leq \lambda_{|N|}(G) \leq \lambda_{|N|}\left(G^{*}\right)
\end{aligned}
$$

The following proposition is introduced, which can be derived from Refs. $[28,30]$ to further explain the relations of the network-splitting patterns and the changes of the Laplacian spectrum.

Proposition 1: A connected graph $\mathrm{G}$ consists of $\mathrm{n}$ sub-graphs $G_{1}, G_{2} \ldots G_{n}$. Each of them is relatively close-connected. For each sub-graph, its corresponding elements of an eigenvector associated with a near-vanishing eigenvalue are approximately identical.
According to Eq. (4), the quadratic form of $\lambda_{2}$ can be rewritten as follows:

$$
\lambda_{2}=\min \sum_{i, j=1}^{|N|} l_{i, j} x_{i} x_{j}=\min \sum_{i, j=1}^{|N|}\left(x_{i}-x_{j}\right)^{2} \cdot\left|l_{i, j}\right|
$$

where $l_{i, j}$ is the element of $L_{e}$.

Based on the aforementioned theorems and proposition, it is obvious that a well-connected network has a large algebraic connectivity and significant differences exist among all elements in the eigenstructure. Conversely, a splitting part of the network can be spectrally mapped into the eigenstructure with small differences among the corresponding elements. In this paper, this numerical pattern is termed as spectrum segmentation.

In the context of the power systems, the spectrum segmentation of $L_{e}$ would change along with the transmission line

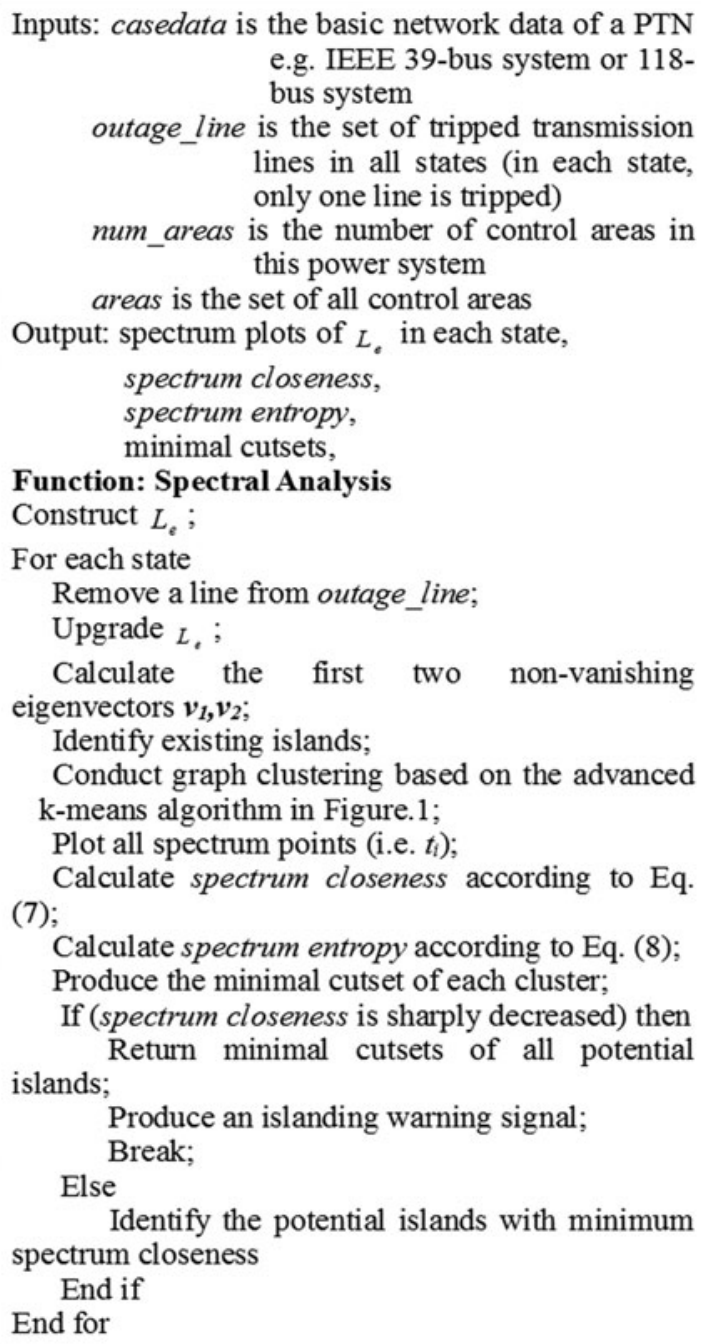

FIGURE 2. Spectral analysis. 


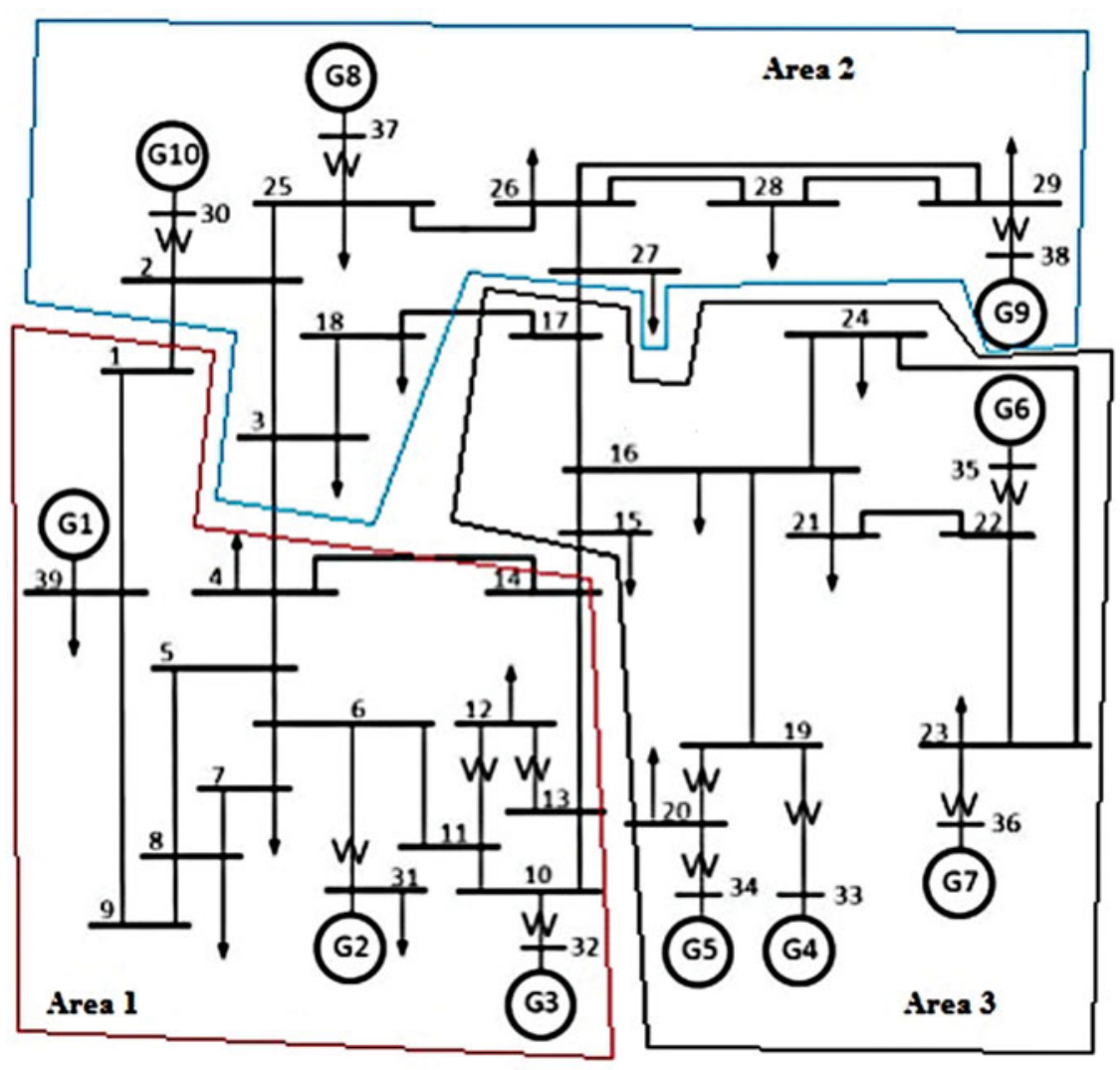

FIGURE 3. The test system based on the IEEE New England 39-bus system.

outages. Clustering vertices into groups depend on the connectivity of the whole network. Based on Theorem 2, any removal of edges in a cluster would weaken the global connectivity while simultaneously increase the dependency between the cluster and the rest of the network. On the other hand, outages of the tie-lines would deteriorate the inter-dependencies of all connected areas. All these conjectures are reflected by the phenomena of spectrum segmentation (e.g., as shown in Figures 2 and 3 ).

In general, $\lambda_{2}$ globally reflects the connectivity of all vertices in a network. Based on the aforementioned analysis, we can safely draw the conclusion that the changes of spectral segmentation in the eigenstructure can naturally reveal the splitting patterns of the network topology. In other words, the coupling level between connected sub-networks can be represented by the level of spectrum segmentation in a spectral domain. For example, in a power transmission network, if the coupling between an area and the rest of the whole network becomes weak, a "spectrum segmentation" would be associated with this area to indicate the topological changes. In the forthcoming subsection, the level of spectrum segmentation is quantified by a newly proposed entropy-based metric.

\subsection{An Improved Spectral Clustering Algorithm}

In this subsection, two proposed metrics (i.e., spectrum closeness (SC) and entropy) are incorporated into a basicnormalized spectral clustering algorithm, which plays an essential role in detecting the topological changes. The two metrics can effectively quantify the process of the integrated power transmission network transiting to separation status.

The basic algorithm of normalized spectral clustering consists of six steps [36]:

(i) Input power adjacency matrix $A_{p}$ and the number $\boldsymbol{k}$ of clusters.

(ii) Compute the normalized electric-Laplacian matrix $L_{e}{ }^{*}:=\boldsymbol{D}^{-1} L_{e}$.

(iii) Compute the first $m$ eigenvectors $v_{l}, \ldots, v_{m}$ of $L_{e}{ }^{*}$ (in this paper, we use $m=2$ for the simplicity of application).

(iv) Construct $\boldsymbol{S} \in|\mathrm{N}| \times m$ containing the first $m$ eigenvectors as columns.

(v) For $i=1,2, \ldots,|\mathrm{N}|$, let $t_{i} \in{ }^{m}$ be the vector corresponding to the $i$ th row of $\boldsymbol{S}$.

(vi) Group the points $\left(t_{i}\right)_{i}=1, \ldots, / N /$ with the k-means algorithm into clusters $\mathbf{C}_{1}, \ldots, \mathbf{C}_{\mathrm{k}}$. 
The core of this application is step (vi); a reasonable clustering can well reveal the connection status of power transmission networks. In Refs. [28, 37], the Euclidean or angular distance (i.e., the angle difference between two vectors stemming from the same origin in a $d$-dimensional space) has been introduced as a metric to quantitatively measure eye-inspected clusters (i.e., all points $t_{i}$ mentioned in step (vi)). Some useful conclusions inspired by empirical observations indicate that the points associated with two closely connected nodes belonging to the same cluster may not be Euclideanly close, while they are well aligned in the similar direction [37]. Therefore, we use an angular distance in step (vi) as a metric for the k-means algorithm.

With the classical k-means algorithm [38], the clustering results based on angular differences can be very much subject to the selected preference. Furthermore, the classical kmeans also suffers from frequent algorithm failures due to the random initialization of cluster centroids [38]. To overcome these drawbacks, an advanced k-means method is developed in the paper, which features the following two aspects: (i) the original set of centroids is initialized by practical control areas in the power transmission network, and (ii) different angular references are selected in different occasions. The main procedure of this algorithm is shown in Figure 1.

$S C$ is proposed to measure the coupling levels of all clusters. Spectrum entropy (SE) is proposed to measure the uncertainty and complexity of Laplacian spectrum and reveals the trend of network evolution. Two definitions are given below:

Definition 3: Let $\boldsymbol{C}_{\boldsymbol{i}}$ be the set of all vertices in a cluster $i$. $\operatorname{ang}_{j}^{i}$ is the angular distance associated with vertex $j . c_{i}$ is the centroid of cluster $i$, which is obtained by the k-means clustering algorithm. Denoted by $\sigma_{i}$, SC of a cluster $i$ indicates the average deviation of all internal spectra points:

$$
\sigma_{i}=\frac{1}{n_{i}} \sum_{j \in C_{i}}\left\|a n g_{j}^{i}-c_{i}\right\|^{2}
$$

where $n_{i}$ is the number of vertices in cluster $i$.

$\mathrm{SC}$ reflects the degree of independence of a cluster. If $\sigma_{i}$ is getting smaller, it means that the connection between cluster $i$ and remaining parts of the graph is being weakened. Otherwise, the coupling of cluster $i$ and the remaining network is being strengthened.

Definition 4: Assume that a graph $G$ is partitioned into $k$ subgraphs (i.e., clusters). The two-dimensional eigenvector space is equally divided into $b$ angular intervals. SE is defined as follows:

$$
E n=\sum_{i}^{k} \sum_{j}^{b} \frac{z_{i}^{j}}{n_{i}} \ln \frac{z_{j}}{n_{i}}
$$

where $z_{i}^{j}$ is the number of points associated with cluster $i$ lying in the interval $j$.

SE globally reflects the level of spectrum segmentation in the eigenspace, which is an important metric for evaluating the clustering performance with different partitioning cardinalities. To examine the effectiveness of a cardinality $k$, a high SE value represents a large difference among all the spectrum points in the spectral domain, which also means that $k$ is less effective to reveal the network-splitting pattern. Otherwise, a small value of SE represents a high level of spectrum segmentation and therefore suggests an emerging network-separating pattern in the topology domain.

Figure 2 illustrates the main procedure of spectral analysis in a given power transmission network.

In the present approach, SC and SE play a key role in evaluating the clustering performance. Given multiple line outages, there are various choices to form the clustering boundaries and address the different potential separations. In our work, the weights of the branches are assigned as the power deliverability. The proposed spectral method aims to find out the partitions with the minimal cutset, which produces the clusters that are the most susceptible to line outages. With different inputs of $k$, SE quantitatively measures the uncertainties involved in the identification of network separation.

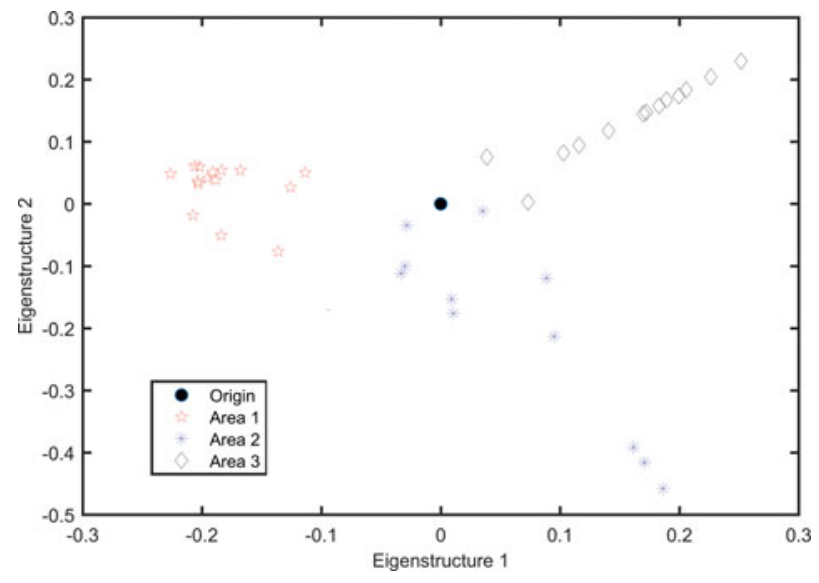

FIGURE 4. Spectrum plot of $L_{e}$ in the original state (New England 39-bus system). 


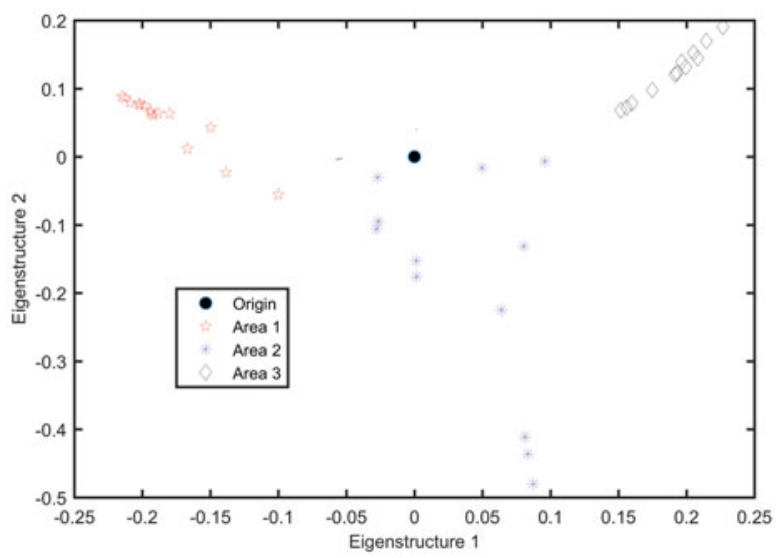

FIGURE 5. Spectrum plot of $L_{e}$ in the post-contingency state (New England 39-bus system).

\section{CASE STUDIES}

In this section, the simulations of islanding detection under two given scenarios are presented. The sequence of cascading failures is determined by the well-established hidden failure model [39] in which the transmission line overload is the main concern. MATLAB serves as the simulation platform. The IEEE New England 39- and 118-bus test systems are used in our case studies.

Normally, an interconnected power system consists of some sub-systems, which are controlled by their respective control entities. Each sub-system is usually termed as a control area. It should be noted that the "control areas" concerned in the case studies are considered as a specific type of network clusters for easy understanding. The proposed approach is feasible to identify the potential network separations with a prior setting of clustering cardinality by which a corresponding $S E$ can be obtained.

\subsection{Case Study on New England 39-Bus System}

Scenario 1 : There are three control areas in this test system, which are shown in Figure 1. The tie-lines are $e_{1,2}, e_{3,4}, e_{14,15}$, and $e_{17,18}$ which are used for connecting different areas. A single line outage of $e_{14,15}$ connecting areas 1 and 3 is considered in this scenario.

Figures 4 and 5 show the spectrum plots of the test system under the initial and post-contingency states, respectively.

As observed in Figures 4 and 5, the spectrum point distribution changes along with the outage of $e_{14,15}$ and the points of cluster 3 are getting closer, which is also reflected by the decrease in SC from 0.2141 to 0.0878 according to Table 1 . Based on the topology in Figure 3 in the pre-contingency state, cluster 3 lost the significant connection to cluster 1 . Accordingly, SE of the whole network is decreased from 0.3442 to 0.3375 in Table 1. On the other hand, SC of cluster 2 is accordingly increased from 0.4996 to 0.5961 , which indicates that the coupling of cluster 2 to the whole network is strengthened. From Table 1, it is obvious that SC of a cluster is significantly decreased if the connection between this cluster and the remainder of the network is getting weaker. In other words, such a cluster is highly vulnerable to separate from the rest of the whole network.

\subsection{Case Study on IEEE 118-Bus Testing System}

Scenario 2: The IEEE 118-bus system is divided into three control areas according to Figure 6. In this scenario, the islanding detection scheme is to be tested for a cascading failure event consisting of five different states, which is based on the mechanism of hidden failure model [40]. In state 1, tie-line $e_{37,40}$ connecting areas 1 and 3 fails due to some unexpected reasons. According to power flow results, this leads to the overload of $e_{37,39}$ and the protection system trips $e_{37,39}$ in state 2 , which is a tie-line of clusters 1 and 3. Due to the outages of $e_{37,40}$ and $e_{37,39}$, the rest of the tie-lines connecting areas 1 and 3 including $e_{38,65}, e_{34,43}$, and $e_{24,70}$ are getting burdened and eventually suffer from overloading. As such, these lines undergo cascading outages resulting in system states 3-5.

Figures 7-9 show the spectrum plots of the test system in different states. Due to the transmission line outages, the connectivity of the whole network deteriorates progressively. As compared with the initial state, the spectrum points of

\begin{tabular}{|c|c|c|c|c|}
\hline State & & Cluster No. and its Vertices & $\mathrm{SC}$ & SE \\
\hline original & $\begin{array}{l}1 \\
2 \\
3\end{array}$ & $\begin{array}{r}14567891011121314313239 \\
23182526272829303738 \\
15161719202122232433343536\end{array}$ & $\begin{array}{l}0.2379 \\
0.4996 \\
0.2141\end{array}$ & 0.3442 \\
\hline Post-contingency & $\begin{array}{l}1 \\
2 \\
3\end{array}$ & $\begin{array}{r}14567891011121314313239 \\
2317182526272829303738 \\
151619202122232433343536\end{array}$ & $\begin{array}{l}0.2438 \\
0.5961 \\
0.0874\end{array}$ & 0.3375 \\
\hline
\end{tabular}

TABLE 1. Comparison of different states (IEEE 39-bus system). 


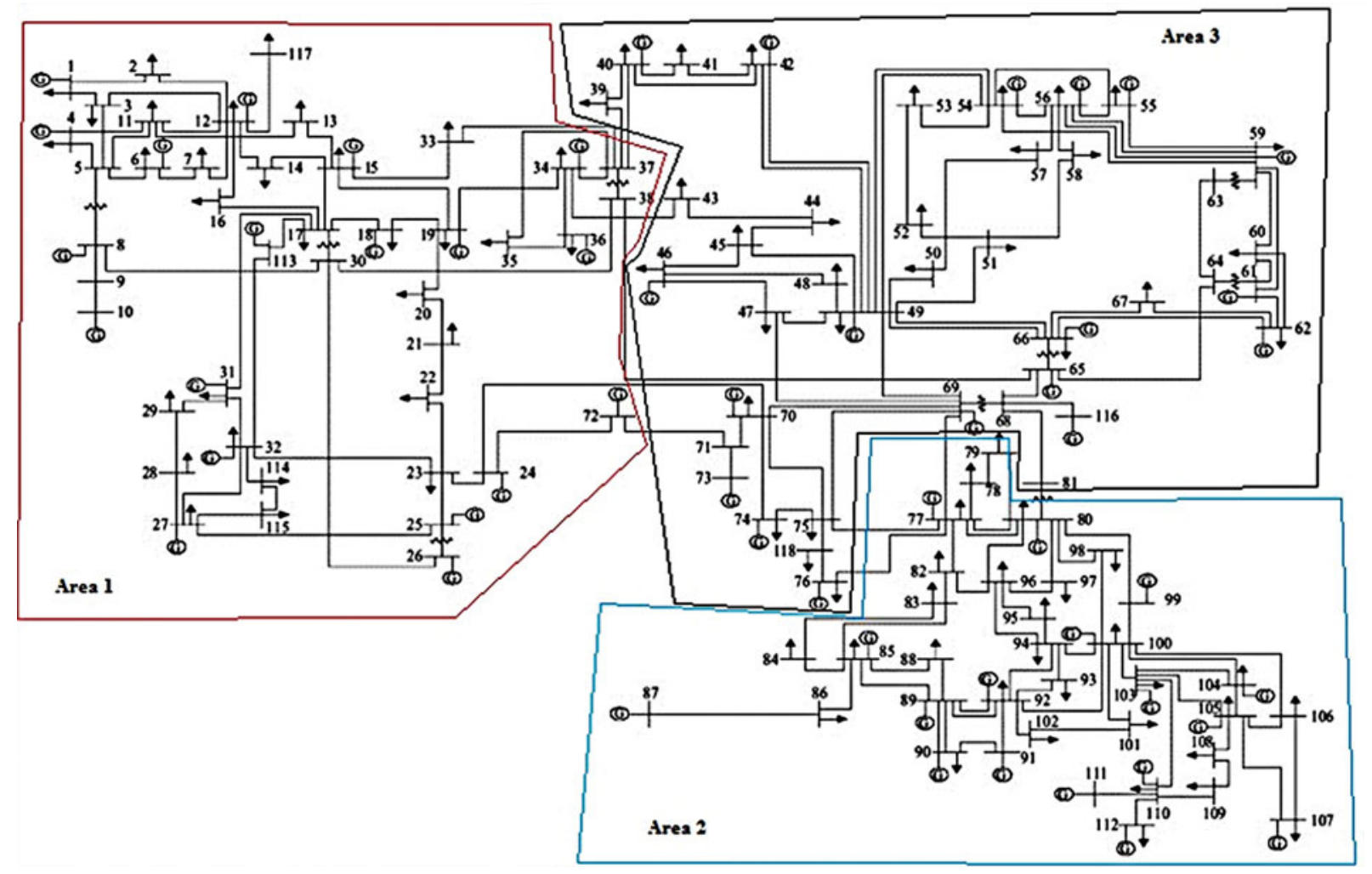

FIGURE 6. IEEE 118-bus test system.

cluster 1 in state 2 are distributed closely under the outages of $e_{37,40}$ and $e_{37,39}$, which are shown in Figures 7 and 8. After the outages of $e_{38,65}, e_{34,43}$, and $e_{24,70}$, the spectrum points of cluster 1 are visually aligned into approximately one straight line in Figure 7, indicating that the islanding formation of cluster 1 is imminent and accordingly, a warning signal can be issued. At the same time, it is also observed

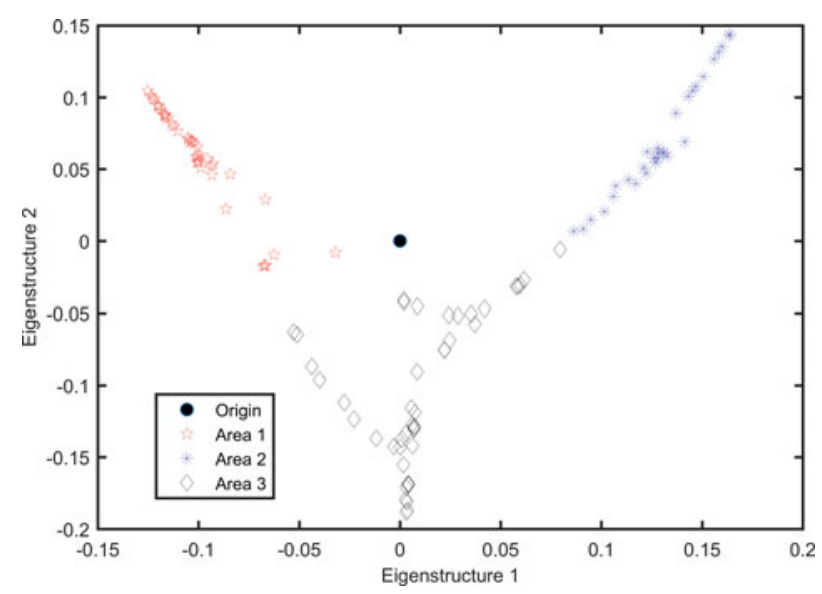

FIGURE 7. Spectrum plot of $L_{e}$ in the original state (IEEE 118-bus system). in Figure 7 that the spectrum points of clusters 2 and 3 disperse significantly. From Table 2, SC of cluster 1 in state 2 as compared to the original state is decreased from 0.3124 to 0.1418 , which indicates that the independence of cluster 1 is notably increased. Along with further failures, SC of cluster 1 is consequently decreased to 0.0882 in state 5 , which echoes the visual observation in Figure 7 and reflects

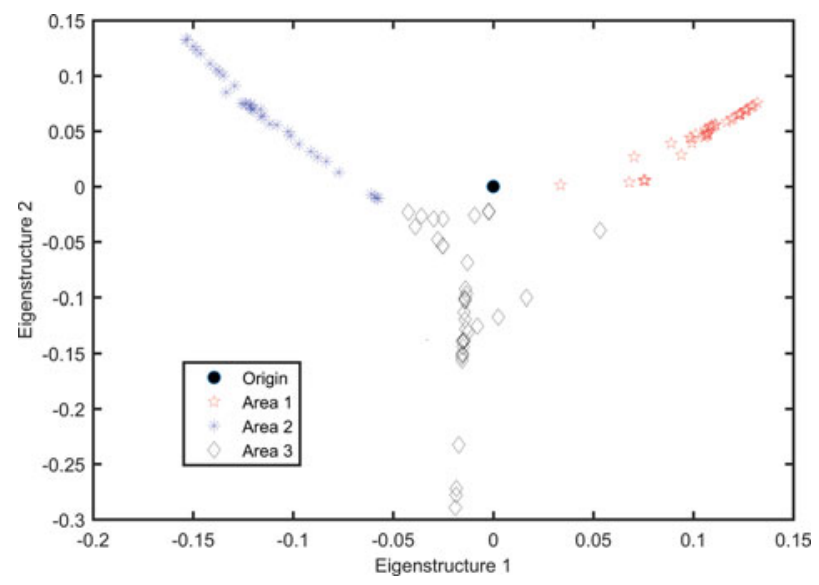

FIGURE 8. Spectrum plot of $L_{e}$ in state 2 (IEEE 118-bus system). 


\begin{tabular}{|c|c|c|c|c|c|}
\hline State & & Cluster No. and corresponding Vertices & $\mathrm{SC}$ & $\sum_{j}^{b} \frac{z_{i}^{j}}{n_{i}} \operatorname{In} \frac{z_{j}}{n_{i}}$ & $\mathrm{SE}$ \\
\hline original & 1 & $\begin{array}{c}123456789101112131415161718192021 \\
222324252627282930313233343536373872 \\
113114115117 \\
777879808283848586878889909192939495 \\
96979899100101102103104105106107108109110 \\
111112 \\
394041424344454647484950515253545556 \\
575859606162636465666768697071737475 \\
7681116118\end{array}$ & 0.3124 & 0.1269 & 0.3592 \\
\hline 1 & $\begin{array}{l}2 \\
3\end{array}$ & $\begin{array}{c}123456789101112131415161718192021 \\
222324252627282930313233343536373872 \\
113114115117 \\
8283848586878889909192939495969899100 \\
101102103104105106107108109110111112 \\
394041424344454647484950515253545556 \\
575859606162636465666768697071737475 \\
76777879808197116118\end{array}$ & $\begin{array}{l}0.1789 \\
0.4508\end{array}$ & $\begin{array}{l}0.0623 \\
0.1657\end{array}$ & 0.3360 \\
\hline 2 & $\begin{array}{l}2 \\
3\end{array}$ & $\begin{array}{c}123456789101112131415161718192021 \\
222324252627282930313233343536373872 \\
113114115117 \\
77787980828384858687888990919293949596 \\
979899100 \\
101102103104105106107108109110111112 \\
394041424344454647484950515253545556 \\
575859606162636465666768697071737475 \\
7681116118\end{array}$ & 0.3261 & 0.0914 & 0.2631 \\
\hline 3 & 3 & $\begin{array}{c}123456789101112131415161718192021 \\
22232425262728293031323334353637384372 \\
113114115117 \\
777879808283848586878889909192939495 \\
96979899100101102103104105106107108109110 \\
111112 \\
3940414244454647484950515253545556 \\
575859606162636465666768697071737475 \\
7681116118\end{array}$ & 0.2745 & 0.1090 & 0.2661 \\
\hline 4 & 2 & $\begin{array}{c}123456789101112131415161718192021 \\
222324252627282930313233343536373872 \\
113114115117 \\
777879808283848586878889909192939495 \\
96979899100101102103104 \\
105106107108109110111112 \\
394041424344454647484950515253545556 \\
575859606162636465666768697071737475 \\
7681116118\end{array}$ & 0.2799 & 0.1027 & 0.2414 \\
\hline 5 & 1 & $\begin{array}{c}123456789101112131415161718192021 \\
222324252627282930313233343536373872 \\
113114115117 \\
777879808283848586878889909192939495 \\
96979899100101102103104105106107108109110 \\
111112 \\
394041424344454647484950515253545556 \\
575859606162636465666768697071737475 \\
7681116118\end{array}$ & 0.0882 & 0.1038 & 0.2441 \\
\hline
\end{tabular}

TABLE 2. Comparison of different states (IEEE 118-bus system). 


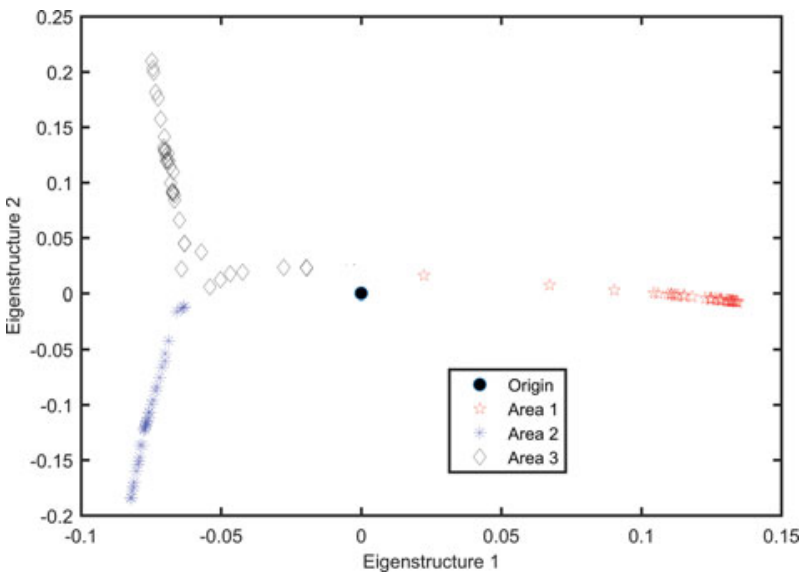

FIGURE 9. Spectrum plot of $L_{e}$ in state 5 (IEEE 118-bus system).

imminent network splitting. In state 5, the minimal cutset of cluster 1 only consists of one edge i.e., $e_{71,72}$. The situation is being critical because the failure of $e_{71,72}$ will eventually cause power system islanding. This analytical situation is consistent with the simulation results as shown in Figure 9 and Table 2 .

\section{CONCLUSIONS}

In this paper, it is aimed to broach the idea that the topological information of the existing or potential network separations in the power systems can be efficiently captured in a spectral domain. A spectral clustering-based approach associated with two evaluation metrics (i.e., $S C$ and $S E$ ) is recently proposed to identify the emerging network separations. Distinguished from the previous methods in this field, the present work has the following two contributions: (i) exhaustive/heuristic search in a whole network is avoided. An NP-hard problem in the topological domain is handled by the proposed approach in the spectral domain, which is computationally efficient; and (ii) the proposed spectral method can be effortlessly extended by considering other electrical features in the construction of the network Laplacians. Case studies on New England 39-bus and IEEE 118-bus systems are carried out. The simulation results demonstrate the effectiveness of the proposed approach.

Admittedly, there are many other factors (e.g., system undamped oscillations, voltage collapse, cascading outages due to transmission line thermal overloads, etc.) that can result in the uncontrolled system separation. The construction of a comprehensive framework that addresses all the necessary factors needs a complex modeling of the power system governing laws and features (e.g., Ohm's law, power flow, line thermal limits, etc.), which will be investigated in our future work.

\section{FUNDING}

This work was partially supported by the Research Grants Council of Hong Kong, China under Grant Nos. T23-407/13N and $\mathrm{T} 23-701 / 14 \mathrm{~N}$.

\section{ORCID}

Chun Sing Lai @ http://orcid.org/0000-0002-4169-4438

Zhao Xu ๑ http://orcid.org/0000-0003-4480-7394

\section{REFERENCES}

[1] Donde, V., Lopez, V., Lesieutre, B., Pinar, A., Chao, Y., and Meza, J., "Identification of severe multiple contingencies in electric power networks," in Power Symposium, 2005. Proceedings of the 37th Annual North American, 2005, pp. 59-66.

[2] Goderya, F., Metwally, A. A., and Mansour, O., "Fast detection and identification of islands in power networks," Power Appar. Syst. IEEE Trans., Vol. PAS-99, pp. 217-221, 1980.

[3] Sasson, A. M., Ehrmann, S. T., Lynch, P., and Van Slyck, L. S., "Automatic power system network topology determination," Power Appar. Syst. IEEE Trans., Vol. PAS-92, pp. 610-618, 1973.

[4] Liacco, T. E. D., and Kraynak, T. J., "Processing by logic programming of circuit-breaker and protective-relaying information,” Power Appar. Syst. IEEE Trans., Vol. PAS-88, pp. 171175, 1969.

[5] Montagna, M., and Granelli, G., "Detection of Jacobian singularity and network islanding in power flow computations," Gener. Transm. Distrib. IEE Proc., Vol. 142, pp. 589-594, 1995.

[6] Di Pierro, F., Khu, S.-T., Savić, D., and Berardi, L., "Efficient multi-objective optimal design of water distribution networks on a budget of simulations using hybrid algorithms," Environ. Modell. Softw., Vol. 24, pp. 202-213, 22009.

[7] Bertran, M., and Corbella, X., "On the validation and analysis of a new method for power network connecticity determination," Power Appar. Syst. IEEE Trans., Vol. PAS-101, pp. 316324, 1982.

[8] Men-Shen, T., "Development of islanding early warning mechanism for power systems," in Power Engineering Society Summer Meeting, 2000. IEEE, Vol. 1, 2000, pp. 22-26.

[9] Pagani, G. A., and Aiello, M., "The Power Grid as a complex network: A survey," Phys. A. Stat. Mech. Appl.

[10] Jeong, H., Tombor, B., Albert, R., Oltvai, Z. N., and Barabasi, A. L., "The large-scale organization of metabolic networks," Nature, Vol. 407, pp. 651-654, October 2000.

[11] Ravasz, E., and Barabasi, A. L., "Hierarchical organization in complex networks," Phys. Rev. E, Vol. 67, February 2003.

[12] Noh, J. D., and Rieger, H., "Random walks on complex networks," Phys. Rev. Lett., Vol. 92, March 2004.

[13] Motter, A. E., and Lai, Y.-C., "Cascade-based attacks on complex networks," Phys. Rev. E, Vol. 66, p. 065102, 12/20/2002.

[14] Lai, Y.-C., Motter, A., and Nishikawa, T., "Attacks and Cascades in Complex Networks," in Complex Networks., Vol. 650, Ben-Naim, E., Frauenfelder, H., and Toroczkai, Z., Eds., ed: Springer Berlin Heidelberg, 2004, pp. 299-310. 
[15] Jalili, M., "Social power and opinion formation in complex networks," Phys. A. Stat. Mech. Appl., Vol. 392, pp. 959-966, 2/15/2013.

[16] Strogatz, S. H., "Exploring complex networks," Nature, Vol. 410, pp. 268-276, March 2001.

[17] Barabasi, A. L., and Albert, R., "Emergence of scaling in random networks," Science, Vol. 286, pp. 509-512, October 1999.

[18] Crucitti, P., Latora, V., and Marchiori, M., "A topological analysis of the Italian electric power grid," Phys. A. Stat. Mech. Appl., Vol. 338, pp. 92-97, July 2004.

[19] Arianos, S., Bompard, E., Carbone, A., and Xue, F., "Power grid vulnerability: A complex network approach," Chaos, Vol. 19, March 2009.

[20] Ash, J., and Newth, D., "Optimizing complex networks for resilience against cascading failure," Phys. A. Stat. Mech. Appl., Vol. 380, pp. 673-683, 7/1/2007.

[21] Watts, D. J., and Strogatz, S. H., "Collective dynamics of 'small-world' networks," Nature, Vol. 393, pp. 440-442, June 1998.

[22] Girvan, M., and Newman, M. E. J., "Community structure in social and biological networks," Proceedings of the National Academy of Sciences of the United States of America, Vol. 99, pp. 7821-7826, June 2002.

[23] Newman, M. E. J., "Modularity and community structure in networks," Proceedings of the National Academy of Sciences of the United States of America, Vol. 103, pp. 8577-8582, June 2006.

[24] Newman, M. E. J., "Detecting community structure in networks,” Eur. Phys. J. B, Vol. 38, pp. 321-330, March 2004.

[25] Radicchi, F., Castellano, C., Cecconi, F., Loreto, V., and Parisi, D., "Defining and identifying communities in networks," Proc. Natl. Acad. Sci. U. S. A., Vol. 101, pp. 2658-2663, March 2004.

[26] Wu, F., and Huberman, B. A., "Finding communities in linear time: a physics approach,” Eur. Phys. J. B, Vol. 38, pp. 331-338, March 2004.

[27] Reichardt, J., and Bornholdt, S., "Detecting fuzzy community structures in complex networks with a Potts model," Phys. Rev. Lett., Vol. 93, November 2004.

[28] Capocci, A., Servedio, V. D. P., Caldarelli, G., and Colaiori, F., "Detecting communities in large networks," Phys. A. Stat. Mech. Appl., Vol. 352, pp. 669-676, July 2005.

[29] Wang, X., and McDonald, J. R., Modern Power System Planning. McGraw-Hill Companies, 1994.

[30] Mohar, B., and Alavi, Y., "The Laplacian spectrum of graphs," Graph Theo. Comb. Appl., Vol. 2, pp. 871-898, 1991.

[31] "Engineering graph clustering: Models and experimental evaluation," J. Exp. Algorithm., Vol. 12, pp. 1-26, 2008.

[32] Wang, H., The Eigenvalues and Laplacian Eigenvalues of $A$ Graph, 2009.

[33] Brouwer, A. E., and Haemers, W. H., "A lower bound for the Laplacian eigenvalues of a graph - Proof of a conjecture by Guo," Linear Algeb. Appl., Vol. 429, pp. 2131-2135, October 162008.

[34] Li, H.-H., Li, J.-S., and Fan, Y.-Z., "The effect on the second smallest eigenvalue of the normalized Laplacian of a graph by grafting edges," Linear Multilin. Algeb., Vol. 56, pp. 627-638, 2008.
[35] Tian, G.-X., and Huang, T.-Z., A New Upper Bound on the Largest Laplacian Eigenvalue of Weighted Graphs, 2008.

[36] Luxburg, U., "A tutorial on spectral clustering," Stat Comp, Vol. 17, pp. 395-416, 12/01 2007.

[37] Donetti, L., and Munoz, M. A., "Detecting network communities: a new systematic and efficient algorithm," J. Stat. Mech. Theo. Exp., Vol. 2004, p. P10012, 2004.

[38] Wilpon, J. G., and Rabiner, L. R., "A modified k-means clustering-algorithm for use in isolated work recognition," IEEE Trans. Acoust. Speech Signal Process., Vol. 33, pp. 587594, 1985.

[39] Chen, J., and Thorp, J. S., "A reliability study of transmission system protection via a hidden failure DC load flow model," in Power System Management and Control, 2002. Fifth International Conference on (Conf. Publ. No. 488), 2002, pp. 384-389.

[40] Phadke, A. G., and Thorp, J. S., "Expose hidden failures to prevent cascading outages [in power systems]," Comp. Appl. Power IEEE, Vol. 9, pp. 20-23, 1996.

\section{BIOGRAPHIES}

Youwei Jia received his B.Eng and Ph.D degrees from Sichuan University, China, in 2011, and The Hong Kong Polytechnic University, Hong Kong, in 2015, respectively. He is now with The Hong Kong Polytechnic University as a postdoctoral fellow. His research interests include power system security analysis, cascading failures, complex network and artificial intelligence application in power engineering.

Chun Sing Lai was born in Staffordshire, U.K. He received the B.Eng. (1st Class Hons.) degree in electrical and electronic engineering from Brunel University London, U.K. in 2013. He is currently working toward the D.Phil. degree in engineering science at the University of Oxford, U.K and is also a research associate at the School of Automation, Guangdong University of Technology, Guangzhou, China. He is a visiting Ph.D. student at The Hong Kong Polytechnic University, Hong Kong, China. His current interests are data analytics and energy economics for renewable energy and storage systems.

Zhao Xu is a Professor with The Hong Kong Polytechnic University, where he is currently Leader of Smart Grid research. He was previously with the Centre for Electric Power and Energy, Technical University of Denmark. He is currently serving as Chairman of IEEE PES Joint Chapter, IEEE Hong Kong Section. He is an Editor, IEEE Transactions on Smart Grid and IEEE Power Engineering Letter. He is also an Editor of Electric Power Components and System Journal. His research interests include grid integration of renewable energies and EVs, electricity market planning and management, and big data and AI applications in power engineering. 